\title{
Chronische Müdigkeit nach Herzinfarkt
}

\author{
Fatigue wird zu wenig beachtet und gegengesteuert.
}

Chronische Müdigkeit - Fatigue - ist eine der häufigsten Begleiterscheinungen chronischer Erkrankungen und die häufigste Ursache für Einschränkungen im täglichen Leben und der Lebensqualität. Chronische Müdigkeit beeinträchtigt die körperlichen, kognitiven, emotionalen und sozialen Funktionen. Patienten mit chronischer Herzinsuffizienz erleben Fatigue als beherrschendes und belastendes Symptom, das zu Unsicherheit führt.

In der Forschung unterscheidet man zwischen fünf Komponenten von Fatigue: allgemeine Fatigue, physische Fatigue, geistige Fatigue, reduzierte Motivation und reduzierte Aktivität. Müdigkeit wird als Anpassungsmechanismus auf Stressoren während der Alarmphase des allgemeinen Anpassungssyndroms (general adaption syndrome $=$ GAS) angenommen. Alsén et al. untersuchten in einem schwedischen Regionalkrankenhaus die Bedeutung von Fatigue auf Patienten mit einem frischen Myokardinfarkt und die Mechanismen mit dem Symptom umzugehen.

\section{Die Ausgangslage}

Ungewöhnliche Fatigue wird bei Patienten mit Herzerkrankungen häufig berichtet, aber auch als häufigstes Symptom vor einem akuten Myokardinfarkt gesehen. Das Konzept der „vitalen Erschöpfung“ geht davon aus, dass ungewöhnliche Fatigue, erhöhte Irritierbarkeit und Gefühle der Enttäuschung das Risiko für einen Myokardinfarkterhöhen. Depressionistweitverarbeitet bei Patienten, die sich von einem Myokardinfarkt erholen und ist auch ein Prognosefaktor für die post-MI Mortalität.

\section{Die Ergebnisse}

Die Befragung mittels Interviews von 19 Personen mit Fatigue vier Monate nach MI ergab als zentrales Thema Leben mit unverständlicher Fatigue, die sich aus einer anderen Qualität von Müdigkeit, unbekannter Ursache, unvorhersehbarem Auftreten und unabhängig von Lösungsversuchen zusammensetzt. Die Folge sind Einschränkungen im persönlichen Leben und das Gefühl, eine Niederlage zu erleiden. Die Studienteilnehmer entwickelten unterschiedliche Methoden mit ihrer Fatigue umzugehen - zeitlich limitierte Strategien und eine Kombination unterschiedlicher Maßnahmen. Häufig wurde die Fatigue nicht überwunden sondern kurzfristig nach einer Ruhepause erleichtert. Als häufigste Strategie veränderten die Teilnehmer ihren Wach-Schlaf-Rhythmus. Auch eine Reduktion der Aktivitäten, gute Planung und eine Struktur für die täglichen

Die Rubrik pflege\&wissenschaft erfolgt in Kooperation mit dem Institut für Pflegewissenschaft der Medizinischen Universität Graz.

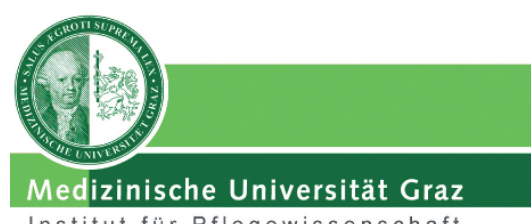

Institut für Pflegewissenschaft

Aufgaben wurden als Anpassungsmechanismen angegeben, ebenso wie die bewusste Überwindung der Fatigue durch Aktivität, Ablenkung oder Vergleich mit Bekannten in ähnlicher Situation und auch eine ausgewogene Ernährung mit Vitaminen, Eisen und Mineralstoffen. Trotz all der unterschiedlichen Maßnahmen konnte die Fatigue nicht dauerhaft bekämpft werden. Die Erleichterung dauerte zumeist nicht lange an und konnte nur durch entsprechende Ruhephasen erleichtert werden.

Fazit

Die Probleme, die Patienten vier Monate nach MI mit Fatigue erleben, sind beträchtlich. Die Patienten fanden es beson- ders irritierend, dass die Ursache für ihre ungewöhnliche Müdigkeit nicht klar erkennbar ist und sie auch keine wirkungsvollen Gegenmaßnahmen setzen können. Da Patienten nach Herzinfarkt auch häufig unter Depressionen leiden, könnte die Fatigue auch ein Teilaspekt der Depression sein, dieser Zusammenhang wurde in dieser Studie nicht untersucht, allerdings brachten die Studienteilnehmer ihre Fatigue in keinen Zusammenhang mit Depression.

In den meisten Fällen verstehen Mitarbeiter des Gesundheitssystems, dass sich Patienten nach einem MI sehr müde fühlen, aber sie haben dieses Problem bisher noch nicht formuliert. Es stellt sich, so die Autoren, die Frage, ob eine frühe Identifikation von Patienten, die nach einem Myokardinfarkt „sehr müde“ sind und eine frühe Intervention eine Verschlechterung des Zustandes verhindern könnte. Pflegekräfte sollten aufmerksam werden, wenn der Patienten feststellt „sehr müde“ zu sein oder ein ungewöhnliches Müdigkeitsgefühl zu haben. Auch der Ausdruck „eine Niederlage zu erleben“ sollte bei Pflegepersonen zu erhöhter Aufmerksamkeit hinsichtlich der möglichen Entwicklung von Fatigue führen. Pflegeinterventionen sollten auf die Identifikation und Reduktion von Stress-auslösenden Faktoren gerichtet sein ebenso wie auf eine verbesserte Fähigkeit des Patienten mit Stress umzugehen. Weitere Untersuchungen sollten sich, so die Autoren, mit der Unterscheidung von Müdigkeit, Fatigue und Erschöpfung sowie auf die Identifikation von Stress-auslösenden Faktoren und nützlichen Lösungsstrategien beschäftigen.

Pia Alsén, Eva Brink \& Lars-Olof Persson: Living with incomprehensible fatigue after recent myocardial infarction, in: JAN, Vol. 64; No 5, Dec. 2008: 459-468
Tab. 1: Patienten, die 15 oder mehr Punkte auf der Multidimensionalen Fatigue-Erfassungs-20 Skala aufweisen $(n=204)$

\begin{tabular}{llllll} 
Geschlecht & $\begin{array}{l}\text { Allgemeine } \\
\text { Fatigue }\end{array}$ & $\begin{array}{l}\text { Körperliche } \\
\text { Fatigue }\end{array}$ & $\begin{array}{l}\text { reduzierte } \\
\text { Aktivität }\end{array}$ & $\begin{array}{l}\text { reduzierte } \\
\text { Motivation }\end{array}$ & $\begin{array}{l}\text { Geistige } \\
\text { Fatigue }\end{array}$ \\
\hline Weiblich & 20 & 19 & 16 & 3 & 11 \\
\hline Männlich & 47 & 46 & 43 & 10 & 27 \\
\hline Gesamt & 67 & 65 & 59 & 13 & 38 \\
\hline
\end{tabular}

